\title{
Políticas públicas e as pesquisas sobre o uso do tempo no enfrentamento das desigualdades de gênero ${ }^{1}$
}

\author{
Public policies and research on the use of time in coping gender inequalities
}

\author{
Tassiane Antunes Moreira ${ }^{2 *}$ \\ Liliane Moser ${ }^{3 * *}$
}

\section{Resumo}

As pesquisas sobre o uso do tempo buscam informações sobre as diversas atividades que os indivíduos realizam ao longo de um determinado período, geralmente 1 dia -24 horas. Com isso, ilustram um retrato do cotidiano das pessoas, abrangendo todas as atividades humanas realizadas. Configuram-se como uma das maneiras de avaliar como o recurso tempo é utilizado de maneira distinta entre homens e mulheres, entre os grupos etários, entre pessoas de variados grupos raciais e de diferentes classes sociais. Este artigo, apoiado em revisão bibliográfica, tem como objetivo central apresentar um breve histórico sobre as pesquisas de uso do tempo no cenário mundial e na América Latina evidenciando as poucas pesquisas quantitativas sobre o uso do tempo realizadas no Brasil e as lacunas que tal processo produz na proposição de políticas públicas direcionadas a equacionar as desigualdades de gênero no País.

Palavras-chave: Uso do tempo. Pesquisas sobre o uso do tempo. Desigualdades de gênero.

\begin{abstract}
Time use surveys look for information about the various activities that individuals perform over a given period, usually one day - 24 hours. With this, they illustrate a picture of the daily life of the people, covering all the human activities carried out. They are one of the ways to evaluate how time is used differently between men and women, between age groups, people of different racial groups and different social classes. This article has as main objective to present a brief history on the research of time use in the world scenario and in Latin America. The few quantitative surveys on the use of time in Brazil and the gaps that such process leaves in the construction of public policies in the country stand out.
\end{abstract}

Keywords: Use of time. Research on the use of time. Inequalities.

\footnotetext{
${ }^{1}$ O presente trabalho foi realizado com apoio da Coordenação de Aperfeiçoamento de Pessoal de Nível Superior (CAPES) - Brasil - Código de Financiamento 001.

2* Assistente Social, Mestre em Serviço Social pela Universidade Federal de Santa Catarina (UFSC). Brasil.2019.

${ }^{3 * *}$ Assistente Social, Doutora em Serviço Social pela Pontifícia Universidade Católica de São Paulo (PUC/SP), Professora do Programa de Pós-graduação em Serviço Social e do Departamento de Serviço Social da Universidade Federal de Santa Catarina (UFSC). Brasil.2019.
} 


\section{Introdução}

O tempo é um bem escasso na dinâmica da vida social moderna. O que chamamos de tempo é o sistema padronizado dos períodos de duração de horas, dias, anos, meses, etc., que se constitui de fato como uma instituição social (ÁVILA, 2004). Conforme explicita Elias (1998), esse "tempo" do qual fala-se e em torno do qual há uma organização, trata-se de uma convenção que regula os períodos de duração dos processos. Nos dias atuais, o tempo é um instrumento de orientação indispensável para realização de uma multiplicidade de tarefas.

Ele não se reduz a um meio de orientação criado pelo homem, mas, além disso, traduz uma instituição cujo caráter varia conforme o estágio de desenvolvimento atingido pelas sociedades. Esclarecer sobre a marcação do tempo, que se materializa no sistema de marcação de horário e calendário, conforme ponderado por Ávila (2004), torna-se interessante para refletir sobre o seu uso no interior das famílias, sobretudo pelas mulheres, e as relações de poder que determinam esse uso.

Partindo-se do pressuposto de que, para a sociedade, a utilização do tempo é definida pelos interesses da acumulação capitalista, entende-se que a categoria "tempo" perpassa as relações de classe e de gênero.

Este artigo tem como objetivo apresentar um breve histórico sobre as pesquisas de uso do tempo no cenário mundial e na América Latina ressaltando as iniciativas do governo brasileiro sobre os estudos relacionados à temática no Brasil e sua importância no sentido de explicitar as diferenças entre homens e mulheres e contribuir na proposição de políticas voltadas a enfrentar as desigualdades de gênero.

Os estudos sobre a maneira como as pessoas, em sua diversidade, alocam os minutos e horas dos seus dias datam de várias décadas. Pesquisadores de alguns países da Europa, assim como na Austrália, Estados Unidos, Canadá e, mais recentemente, de alguns países da América Latina examinam dados resultantes de pesquisas sobre o uso do tempo para analisar como pessoas de diferentes idades, localidades, sexos, inserções profissionais dividem as 24 horas do seu dia em distintas atividades: trabalho, lazer, estudo e cuidado, entre outras. A questão que se revela de maior importância ao se tratar dessa temática diz respeito às diferenças de alocação do tempo entre homens e mulheres (FONTOURA; ARAÚJO, 2016). As desigualdades de gênero observadas no tempo dedicado ao trabalho remunerado e ao trabalho não remunerado é uma realidade visível na maioria 
dos países. Suas causas vêm sendo estudadas e estão relacionadas à questão da reprodução das desigualdades de gênero nas sociedades e às possibilidades de valoração e inserção de homens e mulheres no mercado de trabalho.

Os estudos sobre o uso do tempo trazem elementos importantes para a compreensão de como a divisão sexual do trabalho se traduz no dia a dia das famílias e podem ajudar a compreender as relações entre a sobrecarga de trabalho feminino e a reprodução das desigualdades de gênero.

O acesso a esse tipo de informação abre a possibilidade de confrontar o uso do tempo em duas dimensões: para reprodução econômica e para reprodução social (DEDECCA, 2004). O tempo para reprodução econômica envolve aquele destinado ao trabalho remunerado e gasto com o deslocamento para a sua realização. O tempo para reprodução social e familiar incorpora, por sua vez, basicamente as atividades de organização domiciliar, de lazer e de sono. Ambos os tempos são transformados pelas mudanças econômicas, sociais e culturais.

Na sociedade moderna, a regulação do uso do tempo depende da presença de instituições políticas e culturais. Elas regulam a duração e articulação entre tempo de trabalho remunerado e não remunerado. Assim, não há neutralidade na alocação do uso do tempo. As pessoas realizam a alocação de seu tempo tendo pouca ou relativa autonomia de decisão sobre esse processo.

\section{Breve Caracterização e Histórico das Pesquisas sobre o Uso do Tempo}

Segundo Araya (2003 apud BARAJAS, 2016), as primeiras pesquisas sobre o uso do tempo datam do início do século XX na Europa e nos Estados Unidos e tinham como objetivo realizar estudos demográficos e industriais sobre as populações urbanas e rurais, bem como estudos psicológicos sobre as atividades de lazer das pessoas desempregadas.

Foi a partir dos anos 1970 que as pesquisas de uso do tempo passaram a ser utilizadas com maior frequência pelas feministas, sendo utilizadas com a finalidade de evidenciar a importância do trabalho doméstico não remunerado.Atualmente, em países como a Holanda, Canadá, Dinamarca, Reino Unido e França, as pesquisas sobre o uso do tempo são realizadas periodicamente. Na América Latina, 19 países realizaram pesquisas independentes sobre o uso do tempo ou acrescentaram módulos de perguntas em pesquisas sobre domicílios e seu emprego (BARAJAS, 2016). 
Para Barajas (2016), apesar de os avanços na produção de estatísticas sobre o uso do tempo e trabalho não remunerado terem sido heterogêneos nos diferentes países da América Latina, cabe ressaltar que os legisladores e ocupantes de cargos chaves para a tomada de decisões propuseram leis destinadas à coleta de informações utilizando por referência uma série de instrumentos internacionais sobre a matéria.

É importante destacar que se acordou na comunidade internacional considerar o trabalho não remunerado nos instrumentos de direitos humanos das mulheres. Nessa direção, foram feitas distintas recomendações relativas à coleta de informações sobre uso do tempo e trabalho não remunerado.

Em dezembro de 1979, a Assembléia Geral das Nações Unidas adotava a Convenção para Eliminação de Todas as Formas de Discriminação contra a Mulher (CEDAW, na sigla em inglês). Igualdade de direitos, desenvolvimento e paz foram os lemas da década da mulher, instituída pelas Nações Unidas entre 1976 e 1985.

Para Mast-Kirschning, Lehmann e Albuquerque (1979), a adoção da CEDAW em 1979 foi um divisor de águas na história dos direitos femininos. Segundo os autores, a convenção foi um importante passo para o reconhecimento dos direitos da mulher como direitos humanos. A convenção foi pensada, inicialmente, como instrumento de ajuda ao desenvolvimento. Assim, em seus primórdios, existiam grandes lacunas na proteção dos direitos da mulher.

A situação só mudou de forma duradoura após a Conferência Mundial sobre Direitos Humanos de Viena, realizada em 1993, quando as mulheres passaram de requerentes às titulares de direito. Seguiu-se então à Conferência Mundial sobre a Mulher (Pequim, 1995), que consolidou ainda mais tal compreensão.

A convenção é constituída por um preâmbulo e 30 artigos, 16 dos quais contemplam exigências para que os Estados garantam o respeito aos direitos da mulher. $O$ comitê da CEDAW, formado por 23 peritas, reúne-se em 3 vezes ao ano, Genebra ou Nova York para avaliar os relatórios nacionais dos 186 países, denominados de Estados-Partes, que ratificaram a convenção da ONU (MAST-KIRSCHNING; LEHMANN; ALBUQUERQUE, 1979).

A CEDAW inclui recomendações para quantificar o trabalho não remunerado, realizado majoritariamente por mulheres e meninas. A Recomendação Geral 16 (CEDAW, 1991) assinala que o trabalho não remunerado é uma forma de exploração das mulheres. 
Nesse âmbito, recomenda que os Estados-Partes reúnam dados estatísticos sobre as mulheres que trabalham sem receber remuneração, sem acesso à seguridade social e às contribuições sociais, e recomenda que se adotem medidas para garantir-lhes remuneração, seguridade social e contribuições sociais.

Na mesma direção, a Recomendação Geral 17, que diz respeito à medição e quantificação do trabalho doméstico não remunerado recomenda aos Estados-Partes que fomentem e apoiem pesquisas e estudos experimentais destinados a medir e valorar o trabalho doméstico não remunerado das mulheres.

Da mesma forma, o objetivo estratégico H3 da Plataforma de Ação de Pequim (ONU, 1995) estabelece uma série de medidas a serem adotadas pelos serviços de estatísticas nacionais, regionais e internacionais e pelos órgãos governamentais e organismos das Nações Unidas, com o objetivo de desenvolver informações mais precisas sobre todas as formas de trabalho, a saber:

1. Melhorar a reunião de dados relativos ao trabalho não remunerado que estejam incluídos no Sistema de Contas Nacionais das Nações Unidas, por exemplo, na agricultura, especialmente na agricultura de subsistência, e em outros tipos de atividades de produção que não são de mercado.

2. Desenvolver uma classificação internacional de atividades para as estatísticas sobre uso do tempo, na qual sejam avaliadas as diferenças entre mulheres e homens, em relação ao trabalho remunerado e não remunerado, e reunir dados desmembrados por sexo.

3. Medir quantitativamente o trabalho não remunerado que não é incluído nas contas nacionais e buscar aprimorar os métodos, no intuito de analisar seu valor e indicá-lo, com precisão, em contas-satélites ou outras contas oficiais, elaboradas em separado das contas nacionais básicas, embora em consonância com estas (ONU, 1995).

Barajas (2016) também cita medidas tomadas pela Comissão da Condição Jurídica e Social da Mulher ${ }^{4}$, a qual também expressou consideração pelo trabalho não remunerado realizado pelas mulheres, suas causas e consequências. Nas conclusões acordadas pela Comissão, reconhece-se que a divisão das responsabilidades resulta em consequências, como a fragilização dos vínculos entre as mulheres e o mercado laboral, ao acesso restrito aos benefícios de seguridade social e a menor disponibilidade de tempo para se dedicar à sua educação e à formação pessoal. Reconhece-se também que o trabalho de cuidado remunerado e não remunerado se configura como uma função social,

\footnotetext{
${ }^{4}$ A Comissão da Condição Jurídica e Social da Mulher é uma comissão orgânica vinculada ao Conselho Econômico e Social, criado em virtude da resolução no 11 do Conselho, de 21 de junho de 1946. Trata-se do principal órgão intergovernamental dedicado à promoção da igualdade de gênero e ao empoderamento das mulheres (BARAJAS, 2016, p. 25).
} 
e que esta deve ser distribuída de forma igualitária entre mulheres e homens. Além disso, os Estados-Partes são requisitados a realizar avaliações qualitativas e quantitativas do trabalho não remunerado, bem como medir seu valor e seu preço, de maneira que venha a ser considerado na concepção e elaboração de políticas públicas, em estratégias e planos e nos processos orçamentários.

\title{
As Pesquisas sobre o Uso do Tempo na América Latina
}

$\mathrm{Na}$ América Latina alguns países, como México, Equador e Venezuela, já realizaram pesquisas nacionais sobre o uso do tempo ${ }^{5}$. Essas informações são utilizadas para quantificar o tempo dedicado às atividades domésticas, ao trabalho não remunerado de cuidados e ao trabalho voluntário realizados por homens e mulheres, dentro e fora do domicílio. Para Barajas (2016), essas informações foram úteis para identificar as cargas de trabalho não remunerado e os obstáculos enfrentados pelas mulheres com relação à sua inserção no mercado de trabalho.

Com efeito, e referenciando a autora acima citada, as pesquisas sobre o uso do tempo na América Latina se tornaram importantes ferramentas para

\begin{abstract}
Medir o trabalho doméstico e voluntário não remunerado de mulheres e homens no lar, na comunidade e em associações e organizações sem fins lucrativos; medir o trabalho remunerado no setor informal, incluindo o setor dos lares (contemplado nas contas nacionais); e analisar a inter-relação entre as atividades econômicas e não econômicas de mulheres e homens (BARAJAS, 2016, p. 26).
\end{abstract}

Em seus estudos, Barajas (2016) apresenta dados de 19 países da América Latina ${ }^{6}$ detalhando diferentes instrumentos utilizados para coletar informações sobre o uso do tempo e trabalho não remunerado. Em suas análises constatadas Brasil, México e Venezuela foram precursores, embora com diferentes técnicas e instrumentos para coletar as informações. Segundo seus levantamentos, a autora aponta que antes de 2006, 11 países haviam coletado dados sobre esse tipo de informação, desde então, os

\footnotetext{
${ }^{5}$ México: Módulo na Pesquisa Nacional sobre Trabalho, Contribuições e Uso do Tempo - Entaut (1996), Pesquisa nacional sobre o Uso do tempo - Enut (1998, 2002 e 2009); Equador: Módulo em Pesquisa Domiciliar (2004), Pesquisa Uso do tempo (2005), Pesquisas Nacionais de Uso do Tempo (2007, 2010 e 2012); Venezuela: Pesquisa de Uso do Tempo (2008 e 2011) (BARAJAS, 2016).

${ }^{6}$ Países que realizaram pesquisas e/ou módulos sobre uso do tempo e trabalho não remunerado - América Latina e Caribe: Argentina, Bolívia, Brasil, Colômbia, Costa Rica, Cuba, Chile, Equador, El Salvador, Guatemala, Honduras, México, Nicarágua, Panamá, Paraguai, Peru, República Dominicana, Uruguai e Venezuela (BARAJAS, 2016).
} 
resultados obtidos forneceram subsídios aos governos para elaborar políticas públicas focadas em atender às necessidades da população.

No Brasil, desde 1992, a Pesquisa Nacional por Amostra de Domicílios - PNAD contempla perguntas sobre o uso do tempo. Em 2009 foi realizada uma pesquisa piloto, com diários sobre o uso do tempo em 5 estados do país.

Em âmbito mais abrangente, Barajas (2016) aponta que:

O primeiro projeto na região latino-americana, em escala nacional e com extenso número de perguntas, no formato de módulo sobre uso do tempo em pesquisa nacional - Encuesta Nacional de Ingresos y Gastos de los Hogares (Enigh), em português, Pesquisa Nacional de Rendimentos e Gastos dos Domicílios -, foi realizado pelo Instituto Nacional de Estatística e Geografia do México (Inegi), em 1996 (RENDÓN, 2000 apud BARAJAS, 2016).

Antes de 2006, 11 países tinham realizado o levantamento de dados sobre o uso do tempo e trabalho não remunerado. Barajas (2016) considera que há uma diferença significativa apontando o aumento da realização de pesquisas e módulos sobre o uso do tempo nos países da América Latina depois de 2006, período em que o Grupo de Trabalho de Estatísticas de Gênero (GTEG) foi criado na Conferência Estatística das Américas da CEPAL.

O GTEG foi fruto de um trabalho conjunto realizado pela ONU Mulheres, pelo Instituto Nacional de Estatística e Geografia do México (INEGI), pela Divisão de Assuntos de Gênero (DAG) da Comissão Econômica para América Latina e o Caribe (CEPAL) e pelo Instituto Nacional das Mulheres do México (Inmujeres) na Conferência Estatística das Américas (CEA) da CEPAL. A criação do referido grupo foi útil para compartilhar conhecimentos e informações relativas ao levantamento de dados sobre uso do tempo e trabalho não remunerado em diferentes países da América Latina.

Para Barajas (2016), um dos maiores desafios a ser superado, com relação à coleta de informações sobre uso do tempo e trabalho não remunerado, é a comparabilidade entre os países, pois ainda não existe uma metodologia uniforme e consensual para a padronização das informações estatísticas produzidas no mundo.

Nos países da região da América Latina existem diferentes métodos de coleta de dados, assim como diferentes abordagens teóricas e metodológicas para analisar as informações prospectadas.

A fim de facilitar e com o intuito de concretizar o objetivo proposto, em agosto de 2009 foi apresentada a Classificação de Atividades de Uso do Tempo na América Latina 
e no Caribe (CAUTAL), cujo objetivo consiste em contribuir para a harmonização das pesquisas sobre o uso do tempo na região, bem como dispor de informações padronizadas a respeito do trabalho remunerado e não remunerado e as atividades pessoais, considerando a perspectiva de gênero (LUNA; MÁRQUEZ, 2010).

As referências internacionais utilizadas para sua elaboração foram: Declaração e Plataforma de Ação de Pequim, Classificação Internacional de Atividades para Estatísticas sobre o Uso do Tempo (ICATUS ${ }^{7}$ ), de 1997; Consenso de Quito, de 2007; Resoluções da Organização Internacional do Trabalho (OIT), de 2008, e experiências nacionais dos seguintes países: México, Espanha, Estados Unidos, Cuba, Chile, Argentina, Equador, entre outras (LUNA; MÁRQUEZ, 2010).

Um dos princípios básicos da CAUTAL consiste no processo de harmonização de dados estatísticos, o GTEG elaborou o documento "Diretrizes e referências para harmonizar as pesquisas de uso do tempo na América Latina e no Caribe (2010)". Um dos objetivos desse documento é o descrito a seguir:

\begin{abstract}
Dispor de estatísticas padronizadas, com enfoque de gênero, que reconheçam, meçam e visibilizem o trabalho total, remunerado e não remunerado, assim como as atividades pessoais que podem ser realizadas durante as 24 horas do dia ou as 168 horas da semana, no intuito de contribuir para a análise, elaboração e avaliação de políticas públicas, sociais, econômicas, trabalhistas e de gênero (FONTOURA; ARAÚJO, 2016).

1. Manter consistência com o Sistema de Contas Nacionais - SCN, considerando as atividades produtivas incluídas no escopo de produção do SCN e as atividades produtivas não remuneradas que não são consideradas no SCN, assim como as atividades não produtivas e pessoais e;

2. Assegurar a comparabilidade com outras classificações existentes (LUNA; MARQUEZ, 2010).
\end{abstract}

Essa classificação, assim como outras que são utilizadas para a produção de dados estatísticos, é um instrumento que sofre atualizações em razão dos avanços conceituais e metodológicos da produção estatística internacional.

Embora se observe um avanço significativo na coleta de informações sobre uso do tempo nos países da América Latina nos últimos anos, Barajas (2016) considera que é necessário que a produção de informações sobre o tema seja institucionalizada. Nesse sentido, poderão ser formuladas e implementadas políticas públicas que deem suporte para as famílias, sobretudo para as mulheres, diminuindo a carga de trabalho não

\footnotetext{
${ }^{7}$ Sigla em inglês de International Classification of Activities for Time Use Statistics.
} 
remunerado a partir de programas que ofereçam infraestrutura e acesso ao consumo de bens e serviços que facilitem o preparo de alimentos, que proporcionem melhoria nas habitações e facilitem o acesso aos serviços de cuidados de crianças, idosos e doentes, entre outras medidas.

\section{Pesquisas sobre o uso do tempo no Brasil}

No Brasil, a Pesquisa Piloto realizada pelo IBGE sobre o uso do tempo não teve abrangência nacional. Apesar de não haver uma pesquisa nacional específica sobre o tema, a Pesquisa Nacional por Amostra de Domicílio (PNAD), do Instituto Brasileiro de Geografia e Estatística (IBGE), a contar dos anos 2000, passou a coletar dados sobre o tempo despendido no trajeto casa-trabalho e tempo dedicado aos afazeres domésticos, além da jornada de trabalho remunerado. Com base nessas informações, foram produzidos estudos a partir da perspectiva de gênero sobre o tempo dedicado ao trabalho remunerado e tempo dedicado ao trabalho doméstico familiar. Em 2001, o IBGE também realizou um pequeno teste de pesquisa de uso do tempo em alguns bairros do Rio de Janeiro, por meio do Curso de Desenvolvimento de Habilidades em Pesquisa (CDHP), que proporciona treinamento em pesquisa aos funcionários do Instituto.

Segundo informações disponibilizadas por Fontoura e Araújo (2016), desde o ano de 2006 a temática sobre o uso do tempo era um dos 11 temas do Encontro Nacional de Produtores e Usuários de Informações Sociais, Econômicas e Territoriais, organizado pelo IBGE.

Em 2007 o IBGE sediou o Seminário Internacional sobre Pesquisas de Uso do Tempo, realizado em parceria com o Fundo de Desenvolvimento das Nações Unidas para a Mulher (UNIFEM) e com o apoio da Secretaria Nacional de Política para as Mulheres $\left(\mathrm{SPM}^{8}\right)$. O seminário reuniu representantes de institutos de estatística de diferentes

\footnotetext{
${ }^{8}$ A Secretaria de Políticas para as Mulheres (SPM) foi criada em 2003, na gestão do Presidente Luiz Inácio Lula da Silva, com o objetivo de promover a igualdade entre homens e mulheres, e cuja a missão foi de promover políticas públicas para garantir direitos às mulheres brasileiras visando à melhoria da sua qualidade de vida e à diminuição das desigualdades, articulando-se de forma transversal com todo o governo federal (BANDEIRA, PETRURLAN, 2016). Em agosto de 2010, a SPM deixou de ser uma secretaria especial e passou a ter o status de ministério e, em 2015, foi incorporada ao então recém-criado Ministério das Mulheres, da Igualdade Racial e dos Direitos Humanos (MMIRDH), unindo a Secretaria de Políticas de Promoção da Igualdade Racial, a Secretaria de Direitos Humanos e a Secretaria de Políticas para as Mulheres. Em maio de 2016, o presidente interino Michel Temer extinguiu o MMIRDH e atribuiu suas funções ao Ministério da Justiça, que passou a se chamar oficialmente Ministério da Justiça e Cidadania - composto pela Secretaria Especial de Políticas para as Mulheres, pela Secretaria Especial de
} 
países, de organismos internacionais e de gestores públicos para estudar as melhores práticas na obtenção de informações relacionadas às estatísticas sobre o uso do tempo (CAVALCANTI; PAULO; HANY, 2010).

A partir dessa experiência, foi lançada a criação de um grupo interinstitucional dedicado à temática dos estudos sobre o uso do tempo, nominado Comitê Técnico de Estudos de Gênero e Uso do Tempo (CGUT), formado por representantes do Instituto Brasileiro de Geografia e Estatística (IBGE), da Secretaria de Políticas para as Mulheres (SPM) e do Instituto de Pesquisa Econômica Aplicada (IPEA), além de contar com a participação da Organização Internacional do Trabalho (OIT) e da Entidade das Nações Unidas para o Empoderamento das Mulheres (ONU Mulheres).

O CGUT tem as seguintes atribuições:

a) promover a realização de estudos e pesquisas e o desenvolvimento de sistemas de informações estatísticas de gênero e uso do tempo; b) fomentar à elaboração de indicadores sobre as relações de gênero e uso do tempo; c) construir um banco de boas práticas que reflita as experiências de incorporação do enfoque de gênero e dos usos do tempo nos trabalhos desenvolvidos pelos institutos de pesquisa e estatísticas oficiais e pelos organismos governamentais de políticas para as mulheres, nos planos nacional e internacional; d) promover e participar de seminários nacionais e internacionais, possibilitando a troca de experiência nestas áreas temáticas e; e) atuar no desenvolvimento de pesquisas, análises de dados e estudos sobre temas relevantes para a implementação de políticas orientadas para a igualdade de gênero (BANDEIRA; PRETURLAN, 2016).

A criação do CGUT teve o objetivo de dar continuidade às iniciativas de incentivo à temática do uso do tempo no âmbito das políticas públicas, além de impulsionar um debate mais amplo e contribuir para a realização da pesquisa nacional de uso do tempo no Brasil.

Foi a partir da criação do referido Comitê que o IBGE e as demais instituições envolvidas se comprometeram oficialmente com a questão.

Com a intenção de dar continuidade à tarefa de obter mais informações sobre o uso do tempo no Brasil e com o objetivo de realizar testes de metodologia, o IBGE realizou em 2009 uma pesquisa piloto sobre o uso do tempo em 5 estados brasileiros, a saber:

Políticas de Promoção da Igualdade Racial, pela Secretaria Especial de Direitos Humanos e pela Secretaria Nacional de Juventude. Contudo, após outra alteração na estrutura do governo a secretaria passa a ser denominada Secretaria Nacional de Políticas para as Mulheres, a SNPM e passa a ser vinculada à Secretaria de Governo da Presidência da República. Atualmente, no governo do Presidente Jair Bolsonaro (20192022) a Secretaria Nacional de Políticas para Mulheres (SNPM), está vinculada ao Ministério da Mulher, da Família e dos Direitos Humanos. 
Pará, Pernambuco, Distrito Federal, São Paulo e Rio Grande do Sul. A metodologia utilizada foi a de coletor eletrônico e de diários em papel, consistindo essa última em deixá-lo com o informante durante 1 dia para registrar todas as atividades realizadas no período de 24 horas. O diário se assemelha a uma agenda, na qual os entrevistados listam suas atividades realizadas em intervalos de tempo. O uso do coletor eletrônico foi uma estratégia para a coleta de informações, onde, a partir de uma entrevista presencial, foram transcritas informações do diário de papel, além de ser realizado um pequeno questionário sobre a percepção subjetiva do entrevistado a respeito do seu próprio tempo.

Os objetivos gerais propostos pela Pesquisa Piloto de Uso do Tempo, do IBGE 2009/2010, são os descritos a seguir:

1. Conhecer como os brasileiros organizam seu tempo e comparar com a população de outros países;

2. Comparar como as pessoas distribuem seu tempo de acordo com o sexo, idade, cor/raça, lugar onde moram, nível de instrução, tipo de trabalho, tamanho da família, entre outras variáveis;

3. Medir a qualidade de vida das pessoas; saber quanto tempo dedicam ao trabalho, aos estudos, ao lazer; saber de quanto tempo dispõem para interagir com outras pessoas, para informar-se e para os cuidados pessoais;

4. Avaliar quanto tempo as pessoas gastam em deslocamentos de um lugar a outro;

5. Medir o tempo que as pessoas dedicam ao trabalho doméstico;

6. Medir o tempo que as pessoas dedicam ao trabalho voluntário;

7. Medir o trabalho realizado em empresas/governos/instituições e o trabalho realizado nos domicílios (IBGE, 2012).

Já os objetivos específicos para a temática de gênero dizem respeito a:

1. Medir as desigualdades entre homens e mulheres na distribuição do tempo;

2. Medir a divisão sexual do trabalho: diferenças de gênero na distribuição da chamada "economia do cuidado" ou "trabalho reprodutivo" - afazeres domésticos e cuidado de pessoas

3. Avaliar como a maior dedicação das mulheres ao trabalho reprodutivo interfere na possibilidade de realização de outras atividades (IBGE, 2012).

O teste foi a campo numa subamostra do teste da PNAD/Contínua de 2009. A amostra total foi composta por aproximadamente 12 mil domicílios, sendo público-alvo uma pessoa de 10 anos ou mais de idade, por domicílio. A ida a campo aconteceu de outubro a dezembro de 2009. 
A figura a seguir detalha a distribuição da Amostra da PNAD Contínua e da Pesquisa de Uso do Tempo pelas Unidades da Federação.

Figura 1 - Total e participação das Unidades da Federação na Amostra da PNAD Contínua e da Pesquisa do Uso do Tempo ${ }^{9}$

\begin{tabular}{c|c|c|c|c|c|c|c|c}
\hline \multirow{2}{*}{\begin{tabular}{c} 
Unidade da \\
\cline { 2 - 9 }
\end{tabular}} & $\begin{array}{c}\text { Amostra } \\
\text { de } \\
\text { municípios }\end{array}$ & $\begin{array}{c}\text { Amostra } \\
\text { de } \\
\text { setores }\end{array}$ & $\begin{array}{c}\text { Domicilios } \\
\text { por setor }\end{array}$ & $\begin{array}{c}\text { Total de } \\
\text { domicilios }\end{array}$ & $\begin{array}{c}\text { Amostra } \\
\text { de } \\
\text { municipios }\end{array}$ & $\begin{array}{c}\text { Amostra } \\
\text { de } \\
\text { setores }\end{array}$ & $\begin{array}{c}\text { Domicílios } \\
\text { por setor }\end{array}$ & $\begin{array}{c}\text { Total de } \\
\text { domicilios }\end{array}$ \\
\hline PA & 93 & 336 & 14 & 4.704 & 93 & 336 & 6 & 2.016 \\
\hline PE & 146 & 612 & 14 & 8.568 & 127 & 416 & 4 & 1.664 \\
\hline RMRJ & 19 & 462 & 14 & 6.468 & 19 & 462 & 4 & 1.848 \\
\hline SP & 331 & 1.083 & 14 & 15.162 & 262 & 738 & 4 & 2.952 \\
\hline RS & 211 & 699 & 14 & 9.786 & 170 & 475 & 4 & 1.900 \\
\hline DF & 1 & 195 & 14 & 2.730 & 1 & 195 & 8 & 1.560 \\
\hline Total & 801 & 3.387 & - & 47.418 & 672 & 2.622 & - & 11.940 \\
\hline
\end{tabular}

Fonte: Cavalcanti; Paulo; Hany (2010).

A Pesquisa Piloto de Uso do Tempo, do IBGE, ofereceu um panorama sobre as diversas atividades executadas por homens e por mulheres em diferentes municípios brasileiros. Para Bandeira e Preturlan (2016), a pesquisa configurou-se como uma promissora base a partir da qual se criaram expectativas de que novas iniciativas poderiam ser produzidas para a coleta de dados e informações sobre uso do tempo e trabalho não remunerado no Brasil.

Ainda em 2010, fruto dos trabalhos realizados pelo CGUT, foi realizado na cidade do Rio de Janeiro o II Seminário Internacional sobre o Uso do Tempo: Aspectos Metodológicos e Experiências Internacionais. Em 2012, ocorreu em Brasília outro seminário sobre o uso do tempo: Uso do Tempo e Políticas Públicas de Cuidado: reflexões para uma agenda de desenvolvimento sustentável, o qual promoveu reflexões sobre as pesquisas de uso do tempo em diversos países latino-americanos e suas interfaces com as políticas públicas.

Na 34a Conferência Internacional da Associação de Pesquisas de Uso do Tempo $\left(\operatorname{IATUR}{ }^{10}\right)$, que aconteceu entre os dias 22 e 24 de agosto de 2012 no Japão, o CGUT foi representado pelo IBGE e pela SPM. Nessa Conferência foram realizadas 3 apresentações: 2 delas se referiam à pesquisa piloto sobre o uso do tempo realizada pelo IBGE, em 2010, e outra apresentando a política de creches brasileiras e a série histórica de 2001 a 2009

\footnotetext{
${ }^{9}$ A figura 1 traz em seu interior as siglas RM/RJ referindo-se à Região Metropolitana do Rio de Janeiro.

${ }^{10}$ Sigla em inglês de International Association for Time Use Research.
} 
dos dados da Pesquisa Nacional por Amostra de Domicílio (PNAD) (BANDEIRA; PRETURLAN, 2016).

A partir do esforço despendido e do trabalho realizado pelo CGUT, em 2013 o Brasil sediou a 35a IATUR, no Rio de Janeiro, organizada pela Secretaria de Políticas para as Mulheres, pelo Instituto Brasileiro de Geografia e Estatística e pelo Instituto de Pesquisa Econômica Aplicada.

A Conferência tratou de temas relacionados à matéria, conferindo possibilidade de discutir e conhecer dados e o estágio das diversas pesquisas sobre o uso do tempo em inúmeros países do continente. Como resultado da realização da 35a Conferência Internacional da Associação de Pesquisas de Uso do Tempo realizada em 2013 no Brasil, foi publicado em 2016 o livro: "Uso do Tempo e Gênero", organizado por Natália Fontoura e Clara Araújo, o qual apresenta trabalhos e artigos debatidos na Conferência, cujo tema principal foi estruturado em torno da rubrica acadêmica "Uso do Tempo" - tema que, segundo as organizadoras, remete a um campo de estudos interdisciplinares e aglutina uma gama de trabalhos de cunhos estatísticos, sociológicos, demográficos, econômicos e de outras áreas das Ciências Humanas e Sociais.

Com relação às pesquisas sobre o uso do tempo realizadas no cenário internacional, assim como no Brasil, pode-se observar que elas utilizam diversas metodologias de investigação na tentativa de mensurar o tempo gasto no trabalho remunerado e o tempo gasto no trabalho doméstico familiar.

No Brasil, apesar dos avanços, a experiência de pesquisas com diários do uso do tempo ainda é recente e ficou limitada à Pesquisa Piloto do IBGE. Pode-se considerar que esta pesquisa realizada em 2009 e 2010 possui caráter localizado e não teve abrangência nacional, contemplando apenas 5 regiões do país, além das perguntas realizadas referirem-se a um número restrito de atividades.

Para Fontoura et al. (2010), há um consenso de que a produção estatística sobre o uso do tempo no Brasil ainda é insuficiente para subsidiar a formulação de políticas públicas de conciliação entre trabalho e vida familiar e para uma discussão mais aprofundada de metodologias de contabilização do trabalho reprodutivo na economia nacional.

Tendo por base esse breve levantamento sobre as pesquisas de uso do tempo e as iniciativas do governo brasileiro relacionadas à temática no país, pontuamos algumas 
questões que não são levadas em conta, ou seja, que não são apreendidas nas pesquisas de uso do tempo a partir das metodologias instituídas.

As observações são feitas com referência às análises propostas por Bandeira e Preturlan (2016). Para as autoras, as pesquisas de uso do tempo, em geral, tendem a se concentrar na tradicional divisão sexual do trabalho, deixando de levar em consideração informações importantes relativas ao tempo pessoal, ou tempo subjetivo dos indivíduos, incluindo outras dimensões que são diferentemente interpretadas por homens e mulheres e não têm as mesmas implicações segundo o gênero, como os cuidados estéticos com o corpo, o tempo gasto com a prática de exercícios físicos e o tempo utilizado para a prática de esportes, entre outros. Da mesma maneira, não consideram o tempo gasto na relação com os serviços sociais, ou seja, o tempo gasto com o acesso aos serviços públicos de saúde, educação e assistência social.

Face a essas constatações, ressalta-se a importância de incluir nas análises sobre os usos do tempo, a categoria trabalho familiar, proposta por Saraceno e Naldini (2003), uma vez que compreende o conjunto de atividades desenvolvidas pela família no processo de provisão de bem-estar social e abrange as tarefas domésticas; o cuidado de seus membros, especialmente os dependentes e os investimentos que ela tem de fazer no campo das relações com outras instituições que lhe exigem energia, tempo e habilidades.

A definição de trabalho familiar engloba o trabalho doméstico, o trabalho de cuidados para com familiares e dependentes, o trabalho de consumo, que está relacionado à compra e preparação de alimentos, o trabalho para o acesso aos serviços públicos e privados e o trabalho de relação. O trabalho familiar incorpora ainda o gerenciamento

da interface entre indivíduos, família e sociedade, passando pela rede de amigos e parentes, os serviços e o mercado de trabalho (SARACENO; NALDINI, 2003).

Para Mioto (2015), o trabalho familiar, constitui-se uma questão relevante no contexto contemporâneo, sobretudo no campo da política social, com 2 polos de debates em disputa: um vinculado às políticas de seguridade social, que reafirma a lógica de naturalização do trabalho familiar, e outro, por meio de políticas de conciliação entre trabalho e responsabilidades familiares, o qual tende a propor soluções para o conflito entre essas duas esferas. 
A abordagem aqui assumida busca desnaturalizar e dar visibilidade ao trabalho familiar realizado no espaço doméstico, bem como refletir sobre a necessidade de instauração de mecanismos públicos que proporcionem às mulheres e às famílias condições para gerir o trabalho de reprodução social e para enfrentar os problemas advindos da precarização do mundo do trabalho.

Do ponto de vista metodológico e considerando a perspectiva de gênero, alguns tópicos que ainda são escassos ou ausentes nas pesquisas sobre os usos do tempo são apontados por diversos autores que problematizam o tema, a saber:

1. A necessidade de se considerar os percursos sexuados associados aos usos do tempo, evidenciando, por exemplo, que as categorias de idade, raça e/ou cor e sexo não se apresentam como simples variáveis; ao contrário, se articulam com os sistemas de hierarquia e de poder. Exemplificando: as profissões que são feminizadas continuam sendo as mesmas há mais de um século - mulheres empregadas no setor têxtil, servidoras públicas nos setores administrativos, trabalhadoras na saúde, empregadas do comércio e da indústria alimentar, professoras primárias e secundárias, entre outras. Com perfis diversos, as mulheres estão presentes nestas ocupações desde os anos 1920 (BANDEIRA; PRETURLAN, 2016).

2. As mulheres trabalhadoras da área de cuidados são confrontadas com as exigências de um público próprio. A elas são demandadas habilidades específicas. No entanto, muitas daquelas que têm cônjuge e filhas(os) são confrontadas com as exigências postas por sua família. São demandas e tempos diversos. Com isso, são expostas a dois tipos de tensões ou conflitos concomitantes, oriundos dessas múltiplas demandas externas-internas, nem sempre passíveis de ser por elas respondidas a contento (BESSIN e GAUDART, 2009 apud BARAJAS, 2016).

3. No geral, há competências e/ou incompetências e aptidões e/ou inaptidões que são consideradas como naturalizadas para homens e mulheres. Por exemplo: dirigir melhor ou pior; ler um mapa rodoviário; ter mais senso de orientação no trânsito; realizar diversas atividades ao mesmo tempo etc. Nem sempre essas práticas sexualizadas são questionadas ou desconstruídas nas pesquisas; ao contrário, são racionalizadas e incorporadas, quando muito preservando certa neutralidade sobre as relações de gênero (BESSIN e GAUDART, 2009 apud BARAJAS, 2016). Cabe destacar, inclusive, que essas práticas são agravadas quando associadas a práticas racistas.

4. Outro aspecto a se considerar é que estas pesquisas se apoiam amiúde sobre uma noção quantificável e linear de tempo, dificultando apreender a experiência de mulheres e de homens que descompartimentalizam suas atividades em tempos não uniformes e cronológicos. Como romper e captar os usos do tempo que não seja pela perspectiva do tempo uniforme dominante? Dito de outro modo, como ir além da cultura hegemônica do trabalho formal - computável, mensurável, quantificável, linear e monocrômico - , portanto, da hegemonia de um tempo masculino? (BANDEIRA; PRETURLAN, 2016).

5. Isso leva a outro problema sobre as pesquisas de usos do tempo: registrar os tempos de trabalho na esfera da reprodução e dos cuidados com caracterizações constantemente sexuadas. Nessa lógica, solicitude e a atenção em relação ao outro seriam características preponderantemente femininas. Outro exemplo: sexualizar e/ou generizar a maneira como as mulheres relatam suas trajetórias e/ou histórias, com um nível exacerbado de detalhes, menor objetividade etc. Ou ainda, ao considerar que as dimensões lúdicas são masculinas e as altruístas são femininas (BANDEIRA; PRETURLAN, 2016). 
As autoras consideram que "o tempo e seus usos não são neutros, mesmo quando apreendidos na série temporal de diários do uso do tempo, uma vez que a objetividade da coleta não está isenta de sobrevalorizar ou estigmatizar uma ou outra atividade, hierarquizando um sexo frente ao outro" (BANDEIRA; PRETURLAN, 2016, p. 20). Diante dessa constatação, pode-se afirmar que os usos do tempo contribuem para a produção de desigualdades.

Sendo assim, fazem-se necessárias "novas posturas metodológicas que questionem esse sistema hegemônico de sexo e/ou gênero". Sugere-se então, que as pesquisas sobre o uso do tempo devem apreender as pluralidades em suas múltiplas dimensões, sobretudo de forma mais qualitativa com a finalidade de fornecer uma base de informações para elaboração de políticas e ações públicas mais abrangentes e menos estigmatizantes. Nessa direção, "a produção de pesquisas quantitativas mais universalizantes e amplas deve ser combinadas com pesquisas mais qualitativas localizadas e específicas para diferentes segmentos e grupos sociais" (BANDEIRA; PRETURLAN, 2016, p. 20).

Diante do exposto, apresenta-se a necessidade de se aprofundar esse debate no campo no Serviço Social, uma vez que os assistentes sociais têm sido historicamente os agentes profissionais responsáveis pela implementação das políticas sociais, especialmente as políticas públicas.

\section{Considerações Finais}

Este artigo teve como objetivo apresentar um histórico sobre as pesquisas de uso do tempo no cenário mundial, com ênfase nos estudos realizados sobre a temática no Brasil.

As pesquisas sobre o uso do tempo no cenário internacional vêm se consolidando como instrumentos valiosos para elaboração de indicadores das condições de vida da população. Estas pesquisas fornecem dados relevantes, capazes de subsidiar políticas públicas.

No Brasil, as poucas pesquisas quantitativas sobre a temática possuem caráter localizado e deixam lacunas no processo de construção e estruturação de políticas públicas. 
Diante da conjuntura de desmonte de várias políticas públicas no atual governo brasileiro, percebe-se que essa discussão parece não ter relevância. Podemos citar a polêmica em torno da realização do Censo Demográfico 2020 que está ameaçada. Sem verba, o Instituto Brasileiro de Geografia e Estatística - IBGE, afirma que a realização do Censo está condicionada à aprovação de um concurso público de reposição do quadro de servidores do Instituto. Em declaração dada à imprensa, a equipe econômica do governo defende a realização de um censo mais enxuto, por conta de restrições orçamentárias.

Percebe-se que há muito trabalho a ser feito. A escassez de recursos humanos no IBGE ameaça o plano de trabalho do órgão e a produção de informações estratégicas para o país. Não realizar o Censo Demográfico traria prejuízos internos e risco à imagem internacional do Brasil, alertou o IBGE. Os indicadores produzidos nas pesquisas orientam investimentos e subsidiam políticas implementadas pelas três esferas de governo, mas para que se tornem instrumentos realmente efetivos, a produção de informações depende de recursos financeiros, tecnológicos e humanos.

\section{Referências}

ÁVILA, M. B. Vida cotidiana e o uso do tempo pelas mulheres. In: CONGRESSO LUSO-AFROBRASILEIRO DE CIÊNCIAS SOCIAIS, 8., 2004, Coimbra. Anais [...]. Coimbra: CLABCS, 2004. p. $1-12$.

BANDEIRA, L. M; PRETURLAN, R. B. As pesquisas sobre o uso do tempo e a igualdade de gênero no Brasil. In: FONTOURA, N.; ARAÚJO, C. Uso do tempo e gênero. Rio de Janeiro: UERJ, 2016. p. 43-57.

BARAJAS, M. de la P. Avanços na América Latina na medição e valoração do trabalho não remunerado realizado pelas mulheres. In: FONTOURA, N.; ARAÚJO, C. Uso do tempo e gênero. Rio de Janeiro: UERJ, 2016. Cap. 1, p. 21-42.

CAVALCANTI, L. G. de A.; PAULO, M. A.; HANY, F. E. S. A pesquisa piloto de uso do tempo do IBGE 2009/2010. In: SEMINÁRIO INTERNACIONAL FAZENDO GÊNERO, 9., 2010, Florianópolis. Florianópolis: UFSC, 2010. p. 1-10. Tema: Diásporas, Diversidades, Deslocamentos.

CEDAW - COMITÉ PARA LA ELIMINACIÓN DE LA DISCRIMINACIÓN CONTRA LA MUJER. Recomendação Geral no 16, décimo período de sessões. 1991. Disponível em: http://www.un.org/womenwatch/daw/CEDAW/recommendations/recomm-sp.htm. Acesso em: 7 dez. 2017.

DEDECCA, C. S. Tempo, trabalho e gênero. In: COSTA, A. A. et al. Reconfiguração das relações de gênero no trabalho. São Paulo: CUT, 2004. Cap. 2, p. 21-52. Disponível em: http://library.fes.de/pdf-files/bueros/brasilien/05632.pdf. Acesso em: 10 set. 2017. 
ELIAS, N. Sobre o tempo. Rio de Janeiro: Jorge Zahar, 1998.

FONTOURA, N. et al. Pesquisas de uso do tempo no Brasil: contribuições para a formulação de políticas de conciliação entre trabalho, família e vida pessoal. Revista Econômica, Rio de Janeiro, v. 12, n. 1, p. 11-46, jun. 2010.

FONTOURA, N.; ARAÚJO, C. Uso do tempo e gênero. Rio de Janeiro: UERJ, 2016.

IBGE. Pesquisa Piloto de uso do tempo 2009: primeiros resultados. In: FÓRUM DO SISTEMA INTEGRADO DE PESQUISAS DOMICILIARES, 12, 2012, Rio de Janeiro. Rio de Janeiro: IBGE, 2012. p. 1-60.

IBGE. Website. 2018. Disponível em: https://www.ibge.gov.br/. Acesso em: 25 mar. 2019.

LUNA, E. G. L; MÁRQUEZ, V. S. Clasificación de actividades de uso del tiempo para América Latina y el Caribe, CAUTAL. In: REUNIÓN INTERNACIONAL ESTADÍSTICAS SOBRE USO DEL TIEMPO Y POLÍTICAS PÚBLICAS, 8., México, DF, 2010. Ciudad México: CEPAL, 2010. Disponível em: https://www.cepal.org/mujer/noticias/noticias/3/40283/cautal.pdf. (Propuesta para discusión). Acesso em: 10 set. 2017.

MAST-KIRSCHNING, U.; LEHMANN, A.; ALBUQUERQUE, C. 1979: Convenção da ONU contra discriminação de mulheres. Revisão de Augusto Valente. Berlin: DW, 1979. Disponível em: http://www.dw.com/pt-br/1979-convenção-da-onu-contra-discriminação-de-mulheres/a5033580. Acesso em: 18 dez. 2017.

ONU - ORGANIZAÇÃO DAS NAÇÕES UNIDAS. Declaração e plataforma de ação de Pequim. 1995. Disponível em: http://www.un.org/womenwatch/daw/beijing/pdf/BDPfA\%20S.pdf. Acesso em: 7 dez. 2017. 